\title{
Single-drill implant induces bone corticalization during submerged healing: an in vivo pilot study
}

\author{
Paolo Trisi, Antonello Falco and Marco Berardini ${ }^{*}$
}

\begin{abstract}
Purpose: The aim of the present paper is to evaluate a simplified implant site preparation technique to preserve bone bulk and enhance osseointegration using a new conical self-tapping implant in cancellous bone.

Materials and methods: Ten Expander ${ }^{\circledR} 3.8 \times 10 \mathrm{~mm}$ implants (NoDrill ${ }^{\oplus}$, Milano, Italy) were inserted in the right side (test group) of sheep's iliac crest using only the pilot drill $1.8 \mathrm{~mm}$ in diameter. Ten $3.8 \times 10 \mathrm{~mm}$ Dynamix ${ }^{\circledR}$ implants (Cortex, Shlomi, Israel) were inserted in the right side (control group) of the same animals following the drilling protocol provided by the manufacturer. Histological, histomorphometric, and biomechanical analyses were performed after 2 months.

Results: Implants that belonged to the test group showed a \%BIC of $70.91 \pm 7.95$ while the control group implants had a \%BIC value of $49.33 \pm 10.73$. The \%BV was $41.83 \pm 6.30$ in the test group and $29.61 \pm 5.05$ in the control group. These differences were statistically significant. A phenomenon of osseocorticalization, characterized by more bone volume percentage around implant area than in the neighboring areas, caused by implant threads geometry, was evident in the test group.

Conclusion: This surgical protocol allows to insert an innovative fixture geometry in low-density bone using only a pilot drill. This technique demonstrated many clinical and histological advantages with respect to standard implant drilling procedures and classical implant geometry.
\end{abstract}

Keywords: Dental implant, Osseocorticalization, Osseointegration, Low-density bone, Bone histology

\section{Introduction}

It is well documented that implant initial bone fixation, known as primary implant stability, represents the prerequisite to achieve a successful long-term osseointegration [1].

Many studies demonstrated that the implant primary stability is strictly influenced by host bone density [2], fixture geometry $[3,4]$, and surgical technique used for preparing bone implant bed [5]. Other studies [6, 7] highlighted that host bone quality and fixture macro geometry as main factors able to influence the primary implant stability.

The implant bone site preparation plays a key role in osseointegration development because it allows to obtain

\footnotetext{
* Correspondence: dottmarcoberardini@gmail.com

Biomaterial Clinical and Histological Research Association, Private Practice, Via Galilei 8, 65122 Pescara, Italy
}

an implant bone bed suitable for the fixture dimensions ensuring primary implant stability.

The excessive surgical trauma prior to implant insertion and the bone temperature rise during standard drilling procedures [8] are other crucial factors modifiable by the surgeon, whose importance is often underestimated.

A minimally traumatic bone drilling is strongly recommended to preserve much bone tissue as possible without impairing its healing potential [9].

To this end, several surgical techniques [10] have been proposed to avoid or reduce bone sacrifice during implant placement procedures to enhance primary implant stability and bone quality.

Some authors suggested to undersize the osteotomic implant site with respect to the implant diameter of 
about $10 \%$ in order to reduce bone cutting and enhance primary implant stability $[11,12]$.

An alternative to implant drilling procedures is represented by the osteotome technique [13] that aimed to compact the bone with the mechanical action of cylindrical steel instruments along the osteotomic walls. This procedure increases the clinical implant success in poor bone density [14] although fractured trabeculae and debris could cause a delay in osseointegration process $[15,16]$.

The osseodensification (OD) technique, recently introduced by Huwais et al. [17], used special burs in noncutting rotation mode in order to move bone inside the osteotomic site instead of removing it. This technique allows to preserve native bone and enhance the bone volume around implants [18] supporting high bone contact with the titanium.

The aim of the present paper is to evaluate a reduced implant site preparation technique to preserve bone bulk and enhance primary stability using a new conical self-tapping implant in cancellous type IV bone. A histologic, histomorphometric, and biomechanical comparative analysis was conducted in lowdensity bone of the sheep's iliac crest. The hypothesis to test is if undersized preparation and compressing implants yield a higher BIC and higher torque and ISQ than standard implant inserted with traditional drilling protocols.

\section{Materials and methods}

The Ethics Committee for Animal Research of the Veterinary School of the University of Teramo (Teramo, Italy) approved the study protocol, which followed the guidelines established by the European Union Council Directive of February 2013 (R.D.53/2013).

Two female sheep, 4-5 years old, were included in the study. Clinical examination determined that all animals were in good general health. Exclusion criteria included general contraindications (pregnancy, systemic disease) to implant surgery and active infection, or severe inflammation in the area intended for implant placement.

The animals were given thiopental (Thiopental, Höchst, Austria) for induction of anesthesia as needed. After oro-tracheal intubation and ventilation, anesthesia was sustained with nitrous oxide oxygen with $0.5 \%$ halothane. Physiologic saline solution was administered during surgery for fluid replacement.

The edges of the iliac crests were exposed through a skin incision of $15 \mathrm{~cm}$ in length. The skin and facial layers were opened and closed separately.

After dissection of the soft tissues, the bone was exposed and five undersized osteotomic sites were prepared in each (left and right) side of the iliac crest.
In the right side of each animal (test group), implant bone sites were prepared using only the pilot drill 1.8 $\mathrm{mm}$ in diameter. Ten Expander $3.8 \times 10 \mathrm{~mm}$ implants (NoDrill ${ }^{\circ}$, Milano, Italy) were inserted in the right side of both animals with a hand control wrench. Maximum insertion torque values were between 45 and $60 \mathrm{~N} / \mathrm{cm}$. In the left side of each animal (control group), implant bone sites were prepared using the following burs sequence: pilot drill $1.8 \mathrm{~mm}$ in diameter, twist drill $2.8 \mathrm{~mm}$ in diameter, and the final drill $3.2 \mathrm{~mm}$ in diameter. Ten $3.8 \times 10 \mathrm{~mm}$ Dynamix $^{\circ}$ implants (Cortex, Shlomi, Israel) were inserted in the left sides of both animals. Maximum insertion torque values were between 30 and $45 \mathrm{~N} /$ $\mathrm{cm}$. The implant drilling procedures were carried out under profuse saline irrigation (1000 rpm). Implants were inserted in cancellous type IV bone.

After implant insertion, cover screws were secured and the surgical wounds were closed by a resorbable periosteal-muscular inner suture, followed by an external cutaneous 2-0 silk suture.

Each animal underwent systemic antibiotic therapy for 5 days with $8 \mathrm{ml}$ long-acting Clamoxil (Pfizer Limited, Sandwich, USA). After surgery, animals received appropriate veterinary care and were allowed free access to water and standard laboratory nutritional support throughout the trial period.

The sheep were sacrificed 2 months after implantation by an overdose of sodium thiopental (Thiopental, Höchst, Austria).

\section{Micromotion analysis}

Bone blocks containing the implants were retrieved from each side of the iliac crest. Each implant was fitted with a one-piece 11-mm straight abutment.

The bone blocks were fixed on a customized loading device to measure implant secondary stability according to a previously described technique [19]. A digital force gauge (Akku Force Cadet, Ametek, Largo, USA) and, on the opposite side, a digital micrometer (Mitutoyo Digimatic Micrometer, Kawasaki, Japan) were used to measure implant micromotion during load application. Horizontal forces of $25 \mathrm{~N} / \mathrm{cm}$ were applied onto the abutment of the implant perpendicularly to the major axis, and the lateral displacement was measured by the digital micrometer $10 \mathrm{~mm}$ above the crest. This parameter represents the "value of the actual micromotion" (VAM) as previously published [20] and validated [21].

\section{ISQ analysis}

Resonance frequency analysis was assessed at the time of animal sacrifice (after 2 months of healing) with the latest Ostell device (Osstell AB, Göteborg, Sweden). The 
implant stability quotient (ISQ), which ranged between 0 and 100 , was recorded.

\section{Removal torque value (RTV) testing}

Removal torque value (RTV) was measured at the time of animal sacrifice (2 months after implantation) after VAM measuring procedures. The RTV was evaluated and recorded for each implant using a digital hand-operated torque wrench (Tonichi STC400CN) by unscrewing the implants until interfacial failure occurred. The digital torque wrench automatically registered the peak removal torque value on the digital display. After the initial interface detachment, the implants were repositioned back in their initial position as accurately as possible and processed for histologic analysis. Although the interfacial detachment created an artifact at the interface, its analysis would still be reliable according to Sennerby et al. [22], who used a similar procedure to study the morphology of the bone-metal rupture.

\section{Histomorphometric analysis}

Specimens were immediately fixed in 10\% neutral buffered formalin and processed for histologic analysis. After dehydration, samples were infiltrated with a methyl-methacrylate resin from a starting solution $50 \%$ ethanol/resin and subsequently $100 \%$ resin, with each step lasting $24 \mathrm{~h}$. After polymerization, the blocks were sectioned and then ground down to about $40 \mu \mathrm{m}$. Toluidine blue staining was used to analyze the different ages and remodeling pattern of the bone. The histomorphometric analysis was performed by digitizing the images from the microscope via a JVC TK-C1380 Color Video Camera (JVC Victor Company, Yokohama, Japan) and a frame grabber. The images were acquired with $\mathrm{a} \times 10$ objective over the entire implant surface. Subsequently, the digitized images were analyzed by the image analysis software IAS 2000 (Delta Sistemi, Roma, Italy).

For each section, the two most central sections were analyzed and morphometrically measured. The histomorphometric parameters calculated were the \% bone-to-implant contact (\% $\mathrm{BIC})$ and the bone volume (\%BV).

\section{Host bone density analysis}

In both iliac crests of each animal, a bone sample was harvested close to the implant sites. The bone samples collected were analyzed in order to establish the bone volume percentage (basal \%BV).

\section{Statistical analysis}

Biomechanical (VAM, RT, and ISQ) and histomorphometrical data (BIC\% and BV\%) of test and control groups were statistically compared by the $T$ test using a dedicated software (GraphPad Prism 6 - www. graphpad.com).

\section{Results}

No implant failure was observed after 2 months of healing. The clinical examination, done immediately after the bone block retrieval, showed no crestal bone resorption. No bone defects around implants, such as fenestration or dehiscence, were detected. The host bone density expressed in bone volume percentage (basal \%BV) was $26.17 \pm 2.35$. This low value of BV\% is common in soft bone, according to the NHS bone classification [23].

Implants that belonged to the test group showed a bone to implant contact percentage (\%BIC) of $70.91 \pm$ 7.95 while the control group implants had a \%BIC value of $49.33 \pm 10.73$. The $\% \mathrm{BV}$ was $41.83 \pm 6.30$ in the test group and $29.61 \pm 5.05$ in the control group (Figs. 1, 2, $3,4,5$, and 6). These histomorphometric parameters

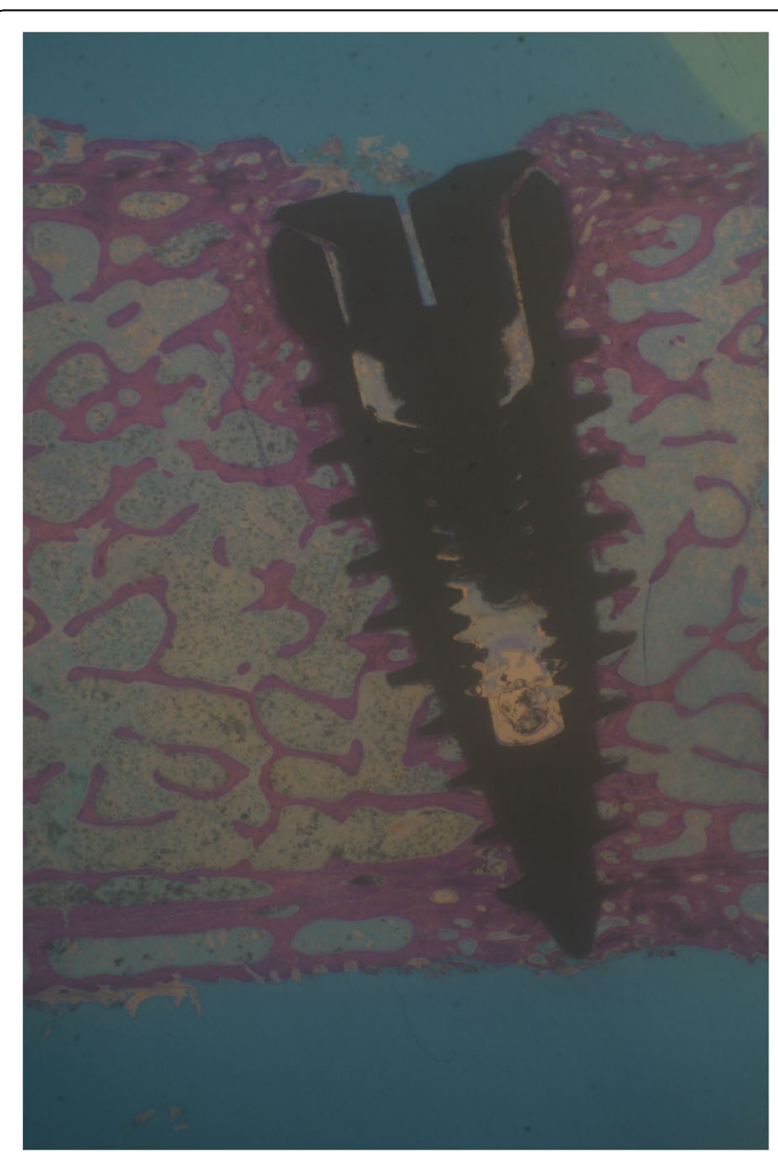

Figure 1 Test group. The implant achieved a high osseointegration degree. The newly formed bone appeared well interconnected with the pre-existing trabeculae. The "corticalization" phenomenon is evident: the bone appears densified around a titanium implant (magnification $\times 8$-toluidine blue) 


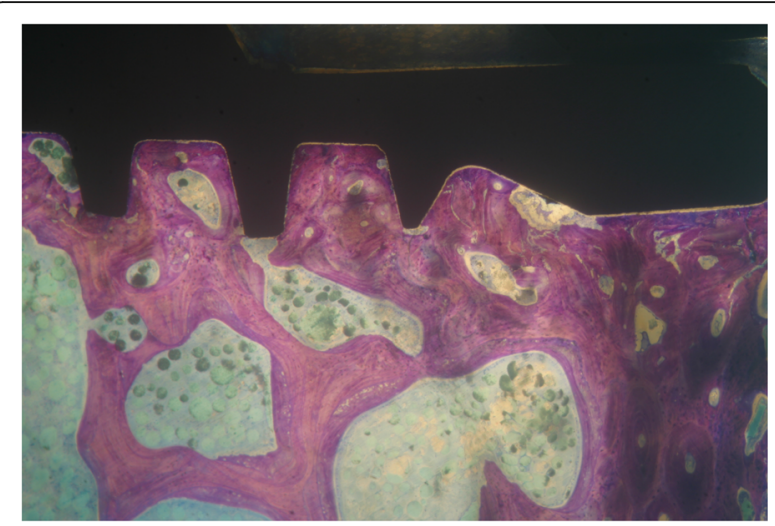

Figure 2 Test group. Implants in the test group showed an extremely high percentage of bone directly contacted to implant surface (magnification $\times 25$-toluidine blue)

were statistically different between the two groups. The statistical comparison between the host bone density (basal \%BV) and \%BV in the test group revealed that expander implants were able to increase in a significant way the host bone density (Tables 1, 2 and 3).

The biomechanical analysis of secondary implant stability revealed a VAM value of $82.6 \pm 23.27$ in the test group and $60.5 \pm 16.58$ in the control group. The reverse torque (RT) was $98.2 \pm 16.81$ in the test group and 98.8 \pm 24.40 in the control one. Histomorphometric and biomechanical data of both groups are summarized in Table 2.

Expander implant surface (test group) was covered by a thick layer of newly formed bone induced by the osteoconduction properties of the implant surface. A considerable amount of fractured trabeculae that led to bone chip condensation was present around implant threads. The osseocorticalization phenomenon characterized by more bone volume percentage around the implant area than in the neighboring

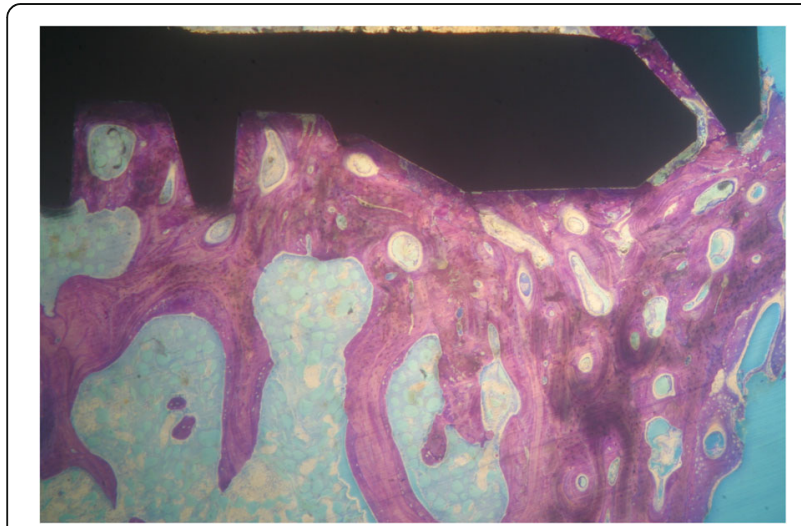

Figure 3 Test group. The present histological photo showed a continuous thin layer of newly formed bone along the neck area of the implant (magnification $\times 25$-toluidine blue)

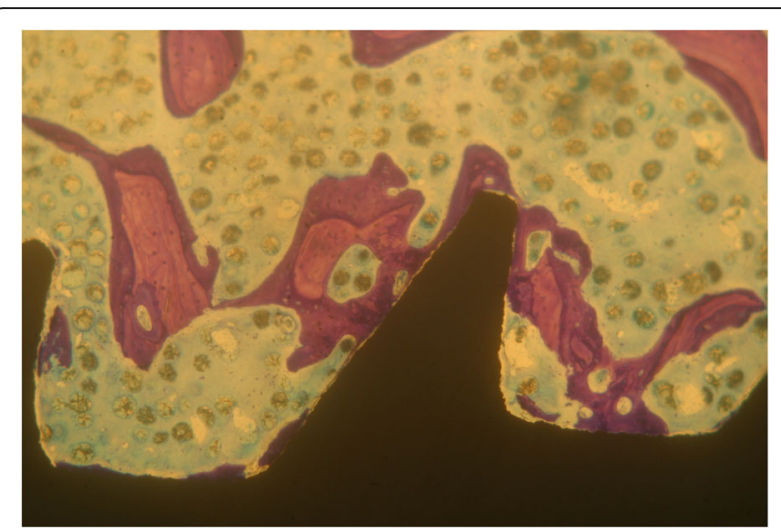

Figure 4 Control group. No bone condensation was possible with traditional burs and standard implant (magnification $\times 25$-toluidine blue)

areas, caused by implant threads geometry, was evident at low magnification. A reparative bone formation process that connected the fractured bone trabeculae to bone fragments and/or to the implant surface was evident, with remodeling phenomena characterized by osteoclastic resorption coupled with osteoid formation.

\section{Discussion}

Results from the present study clearly show that it is possible to insert an implant using a one-step concept for the surgical preparation of the bone bed in cancellous bone.

Guazzi et al. [24], comparing the clinical outcome of implants inserted in sites prepared with a simplified protocol consisting of one large single drill versus multiple conventional drilling steps, demonstrated less surgical time which led to less postoperative morbidity in the test group.

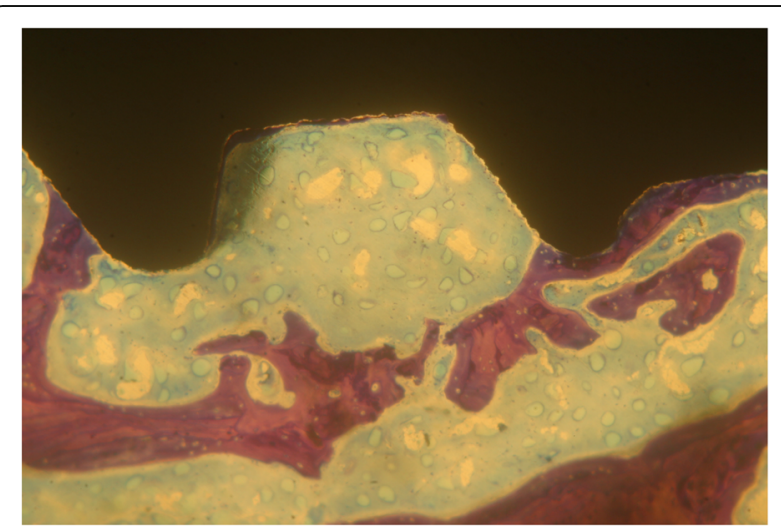

Figure 5 Control group. Implants belonging to the control group showed some small surface areas not contacted with bone (magnification $\times 25$-toluidine blue) 


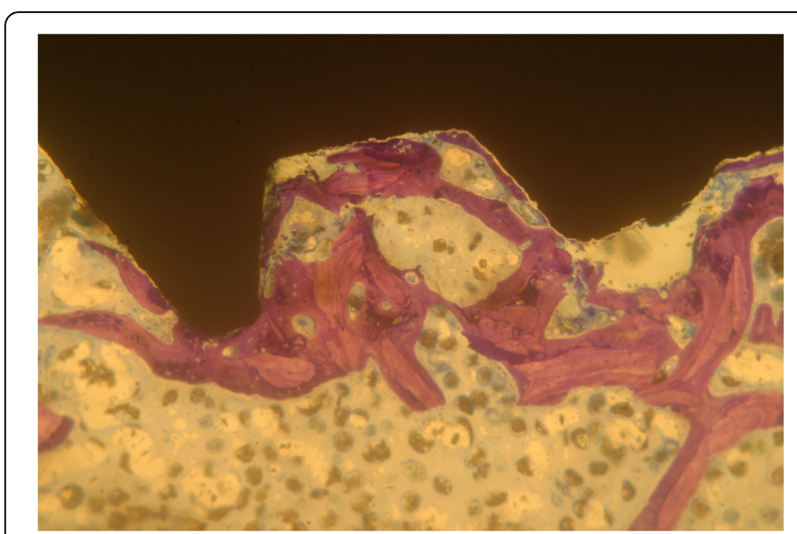

Figure 6 Control group. Some implant thread areas were not covered by bone layer (magnification $\times 25$-toluidine blue)

Gehrke et al. [25] failed in finding differences in implant stability (RFA analysis) between implant inserted with a drill sequence or single large drill.

In the present study, the single-drill protocol was essentially different from that used from previous authors because the osteotomic sites resulted in undersized preparation with respect to the implant diameter. In this way, it is possible to keep more bone inside the implant bed with respect to a large final drill. Also, the significant thermal changes highlighted by some studies [26, 27] when only final large drill is used as single step are avoided by the present surgical protocol which involved the use of only one low-diameter bur.

Undersizing the osteotomic site is a well-documented $[28,29]$ surgical technique that allows to increase primary implant stability in poor bone density. From the biomechanical standpoint, an undersized drilling protocol is demonstrated to be effective in increasing insertion torque in low-density bone [30].

Some authors [31] theorized that a 10\% undersized protocol, in poor density bone, is sufficient to improve the primary stability of the implant.

In the present study, the undersized bone preparation of the implant site of about $2 \mathrm{~mm}$ compared to a 4-mmdiameter implant (almost 100\%) allows to obtain an excellent implant primary stability with insertion torque peaks greater than $45 \mathrm{~N} / \mathrm{cm}$. The implant geometry characterized by self-tapping threads helps the implant body to penetrate into the bone trabeculae without

Table 1 Basal bone volume percentage (basal \%BV) was compared to \%BV around implants after 2 months of healing in both groups. \%BV in the test group was significantly higher than basal \%BV $(P<0.05)$

\begin{tabular}{lll}
\hline Basal \%BV $26.17 \pm 2.35$ & Test group & Control group \\
\hline & $41.83 \pm 6.30^{*}$ & $29.61 \pm 5.05$ \\
\hline
\end{tabular}

*Significant
Table 2 Mean values of histomorphometric parameters (\%BIC and \%BV) and biomechanical values (VAM, reverse torque, and ISQ) of each implant group

\begin{tabular}{|c|c|c|c|c|c|}
\hline $\begin{array}{l}\text { Implant } \\
\text { type }\end{array}$ & $\mathrm{BIC} \%$ & BV\% & $\begin{array}{l}\operatorname{Vam}(\mu \mathrm{m}) \\
\pm \mathrm{SD}\end{array}$ & $\begin{array}{l}\text { Reverse torque } \\
(\mathrm{N} / \mathrm{cm}) \pm \mathrm{SD}\end{array}$ & $\begin{array}{l}\text { ISQ value } \\
\pm S D\end{array}$ \\
\hline $\begin{array}{l}\text { Test } \\
\text { group }\end{array}$ & $\begin{array}{l}70.91 \pm \\
7.95\end{array}$ & $\begin{array}{l}41.83 \pm \\
6.30\end{array}$ & $\begin{array}{l}82.6 \pm \\
23.27\end{array}$ & $98.2 \pm 16.81$ & $\begin{array}{l}63.5 \pm \\
1.30\end{array}$ \\
\hline $\begin{array}{l}\text { Control } \\
\text { group }\end{array}$ & $\begin{array}{l}49.33 \pm \\
10.73\end{array}$ & $\begin{array}{l}29.61 \pm \\
5.05\end{array}$ & $\begin{array}{l}60.5 \pm \\
16.58\end{array}$ & $98.8 \pm 24.40$ & $\begin{array}{l}59.4 \pm \\
1.39\end{array}$ \\
\hline
\end{tabular}

creating excessive bone dust that could delay the osseointegration processes. The high percentage of bone-implant contact (\% BIC), almost double compared to the initial bone density, demonstrates an effective action of bone compaction.

The comparison between the basal \%BV and \%BV in the test group revealed that these innovative implants were able to increase in a significant way the periimplant bone density with respect to starting host bone density. This increased peri-implant bone density extended from about $0.5-0.7 \mathrm{~mm}$ beyond the implant perimeter causing an osseocorticalization around the fixture profile.

A recent study [32] confirmed that the use of the single-drill surgical approach is less invasive and could promote osseointegration. In addition, the accuracy of implant site preparation is better when the single step procedure is used.

\section{Conclusions}

The hypothesis was accepted. Compressing implants with single-drill bone preparation demonstrated many clinical and histological advantages with respect with to standard implant drilling procedures and classical implant shape and design in low-density bone type. It is possible to summarize the advantages of this technique as follows: higher bone to implant contact percentage than the control group (due to the innovative fixture geometry that causes bone compaction), speed of execution (only one-step preparation in low-density bone), high primary implant stability (undersized preparation matched to special fixture shape and

Table 3 Statistical comparison ( $T$ test) of examined parameters between the test and control groups. The histomorphometric analysis demonstrated significant differences in BIC\% and \%BV values between the two implant groups

\begin{tabular}{ll}
\hline BIC\% & $P<0.05$ \\
\hline BV\% & $P<0.05$ \\
Vam & $P<0.05$ \\
Reverse torque & $P>0.05 \mathrm{Ns}$ \\
ISQ & $P<0.05$ \\
\hline
\end{tabular}


thread geometry), high manageable final implant position, high patient comfort, and less cost.

The present pilot study involves only two animals; this limitation could influence the statistical observations we have discussed. Future in vivo studies with a bigger animal numbers or clinical studies with a high sample size are needed in order to confirm the results of the present paper.

\section{Acknowledgements}

The authors wish to thank Cortex ${ }^{\circledR}$ Dental Implants (Shlomi, Israel) and NoDrill ${ }^{\oplus}$ (Milano, Italy) for providing the implants used in the present study.

\section{Authors' contributions}

PT contributed to the study design, animal surgery, and histologic analysis; AF contributed to the animal surgery; MB contributed to the manuscript preparation, data analysis, data collection, and animal surgery. All authors read and approved the final manuscript.

\section{Funding}

No grants were received for the present study.

\section{Availability of data and materials}

All data and materials are available from the corresponding author in Pescara, Italy.

\section{Ethics approval and consent to participate}

The Ethics Committee for Animal Research of the Veterinary School of the University of Teramo (Teramo, Italy) approved the study protocol with the protocol number 8110.

\section{Consent for publication}

The Ethics Committee for Animal Research of the Veterinary School of the University of Teramo (Teramo, Italy) approved the study protocol with the protocol number 8110.

\section{Competing interests}

The authors declare that they have no competing interests.

Received: 30 August 2019 Accepted: 4 December 2019

Published online: 15 January 2020

\section{References}

1. Albrektsson T, Brånemark PI, Hansson HA, et al. Osseointegrated titanium implants. Requirements for ensuring a long-lasting, direct bone-to-implant anchorage in man. Acta Orthop Scand. 1981;52(2):155-70.

2. Molly L. Bone density and primary stability in implant therapy. Clin Oral Implants Res. 2006;2:124-35.

3. Chong L, Khocht A, Suzuki JB, et al. Effect of implant design on initial stability of tapered implants. J Oral Implantol. 2009;35:130-5.

4. Falco A, Berardini M, Trisi P. Correlation Between Implant Geometry, Implant Surface, Insertion Torque, and Primary Stability: In Vitro Biomechanical Analysis. Int J Oral Maxillofac Implants. 2018;33:824-30.

5. Javed F, Romanos GE. The role of primary stability for successful immediate loading of dental implants. A literature review. J Dent. 2010;38:612-20.

6. Marquezan M, Osório A, Sant'Anna E, et al. Does bone mineral density influence the primary stability of dental implants? A systematic review. Clin Oral Implants Res. 2012;23:767-74.

7. Valente ML, de Castro DT, Shimano AC, et al. Analyzing the Influence of a New Dental Implant Design on Primary Stability. Clin Implant Dent Relat Res. 2016;18:168-73.

8. Trisi P, Berardini M, Falco A, et al. Insufficient irrigation induces peri-implant bone resorption: an in vivo histologic analysis in sheep. Clin Oral Implants Res. 2014;25:696-701.

9. Aghvami M, Brunski JB, Serdar Tulu U, et al. A Thermal and Biological Analysis of Bone Drilling. J Biomech Eng. 2018;1:140.

10. Tabassum A, Walboomers XF, Wolke JG, et al. Bone particles and the undersized surgical technique. J Dent Res. 2010;89:581-6.
11. Turkyilmaz I, Aksoy U, McGlumphy EA. Two alternative surgical techniques for enhancing primary implant stability in the posterior maxilla: a clinical study including bone density, insertion torque, and resonance frequency analysis data. Clin Implant Dent Relat Res. 2008;10:231-7.

12. Degidi M, Daprile G. Piattelli A Influence of underpreparation on primary stability of implants inserted in poor quality bone sites: an in vitro study. J Oral Maxillofac Surg. 2015;73:1084-8.

13. Summers RB. A new concept in maxillary implant surgery: the osteotome technique. Compendium. 1994;15:152-62.

14. Summers RB. A new concept in maxillary implant surgery: the osteotome technique. Compendium. 1994:15:152-6.

15. Büchter A, Kleinheinz J, Wiesmann HP, et al. Biological and biomechanical evaluation of bone remodelling and implant stability after using an osteotome technique. Clin Oral Implants Res. 2005;16:1-8.

16. Stavropoulos A, Nyengaard JR, Lang NP, et al. Immediate loading of single SLA implants: drilling vs. osteotomes for the preparation of the implant site. Clin Oral Implants Res. 2008;19:55-65.

17. Huwais S, Meyer EG. A Novel Osseous Densification Approach in Implant Osteotomy Preparation to Increase Biomechanical Primary Stability, Bone Mineral Density, and Bone-to-Implant Contact. Int J Oral Maxillofac Implants. 2017;32:27-36.

18. Trisi P, Berardini M, Falco A, et al. New Osseodensification Implant Site Preparation Method to Increase Bone Density in Low-Density Bone: In Vivo Evaluation in Sheep. Implant Dent. 2016;25:24-31.

19. Trisi P, Perfetti G, Baldoni E, et al. Implant micromotion is related to peak insertion torque and bone density. Clin Oral Implants Res. 2009;20:467-71.

20. Trisi P, Berardini M, Falco A, et al. Effect of Implant Thread Geometry on Secondary Stability, Bone Density, and Bone-to-Implant Contact: A Biomechanical and Histological Analysis. Implant Dent. 2005;24:384-91.

21. Trisi P, Berardini M, Falco A, et al. Validation of value of actual micromotion as a direct measure of implant micromobility after healing (secondary implant stability). An in vivo histologic and biomechanical study. Clin Oral Implants Res. 2016;27:1423-30.

22. Sennerby L, Thomsen P, Ericson LE. A morphometric and biomechanic comparison of titanium implants inserted in rabbit cortical and cancellous bone. Int J Maxillofac Implants. 1992;7:62-71.

23. Rebaudi A, Trisi P, Cella R, et al. Preoperative evaluation of bone quality and bone density using a novel $\mathrm{CT} /$ microCT-based hard-normal-soft classification system. Int J Oral Maxillofac Implants. 2010;25:75-85.

24. Guazzi P, Grandi T, Grandi G. Implant site preparation using a single bur versus multiple drilling steps: 4 -month post-loading results of a multicenter randomised controlled trial. Eur J Oral Implantol. 2015;8:283-90.

25. Gehrke SA, Bettach R, Aramburú Júnior JS, Prados-Frutos JC, Del Fabbro M, Shibli JA. Peri-Implant Bone Behavior after Single Drill versus Multiple Sequence for Osteotomy Drill. Biomed Res Int. 2018;11:2018.

26. Möhlhenrich SC, Abouridouane M, Heussen N, Hölzle F, Klocke F, Modabber A. Thermal evaluation by infrared measurement of implant site preparation between single and gradual drilling in artificial bone blocks of different densities. Int J Oral Maxillofac Surg. 2016:45:1478-84.

27. Lucchiari N, Frigo AC, Stellini E, Coppe M, Berengo M, Bacci C. In Vitro Assessment with the Infrared Thermometer of Temperature Differences Generated During Implant Site Preparation: The Traditional Technique Versus the Single-Drill Technique. Clin Implant Dent Relat Res. 2016;18:182-91.

28. Arosio P, Greco GB, Zaniol T, et al. Sinus augmentation and concomitant implant placement in low bone-density sites. A retrospective study on an undersized drilling protocol and primary stability. Clin Implant Dent Relat Res. 2018;20:151-9.

29. Alghamdi $\mathrm{H}$, Anand PS, Anil S. Undersized implant site preparation to enhance primary implant stability in poor bone density: a prospective clinical study. J Oral Maxillofac Surg. 2011;69:506-12.

30. Stocchero M, Toia M, Cecchinato D, et al. Biomechanical, Biologic, and Clinical Outcomes of Undersized Implant Surgical Preparation: A Systematic Review. Int J Oral Maxillofac Implants. 2016;31:1247-63.

31. Degidi M, Daprile G, Piattelli A. Influence of underpreparation on primary stability of implants inserted in poor quality bone sites: an in vitro study. J Oral Maxillofac Surg. 2015;73:1084-8.

32. Marheineke N, Scherer U, Rücker M, von See C, Rahlf B, Gellrich NC, Stoetzer M. Evaluation of accuracy in implant site preparation performed in singleor multi-step drilling procedures. Clin Oral Investig. 2018;22:2057-67.

\section{Publisher's Note}

Springer Nature remains neutral with regard to jurisdictional claims in published maps and institutional affiliations. 